\title{
Incomer, a DD36E family of Tc1/mariner transposons newly discovered in animals
}

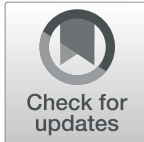

Yatong Sang ${ }^{1 \dagger}$, Bo Gao ${ }^{1,2+}$, Mohamed Diaby ${ }^{1}$, Wencheng Zong ${ }^{1}$, Cai Chen ${ }^{1}$, Dan Shen ${ }^{1}$, Saisai Wang ${ }^{1}$, Yali Wang ${ }^{1}$, Zoltán Ivics ${ }^{2}$ and Chengyi Song ${ }^{1 *}$ (i)

\begin{abstract}
Background: The TC1/mariner superfamily might represent the most diverse and widely distributed group of DNA transposons. Several families have been identified; however, exploring the diversity of this superfamily and updating its classification is still ongoing in the life sciences.

Results: Here we identified a new family of TCl/mariner transposons, named Incomer (IC), which is close to, but distinct from the known family DD34E /TC1. ICs have a total length of about $1.2 \mathrm{~kb}$, and harbor a single open reading frame encoding a $\sim 346$ amino acid transposase with a DD36E motif and flanked by short terminal inverted repeats (TIRs) (22-32 base pairs, bp). This family is absent from prokaryotes, and is mainly distributed among vertebrates (141 species of four classes), including Agnatha (one species of jawless fish), Actinopterygii (132 species of ray-finned fish), Amphibia (four species of frogs), and Mammalia (four species of bats), but have a restricted distribution in invertebrates (four species in Insecta and nine in Arachnida). All ICs in bats (Myotis lucifugus, Eptesicus fuscus, Myotis davidii, and Myotis brandtii) are present as truncated copies in these genomes, and most of them are flanked by relatively long TIRs (51-126 bp). High copy numbers of miniature inverted-repeat transposable elements (MITEs) derived from ICs were also identified in bat genomes. Phylogenetic analysis revealed that ICs are more closely related to DD34E $/ T C 1$ than to other families of Tc1/mariner (e.g., DD34D/mariner and DD $\times$ D/pogo), and can be classified into four distinct clusters. The host and $/ C$ phylogenies and pairwise distance comparisons between RAGI genes and all consensus sequences of ICs support the idea that multiple episodes of horizontal transfer (HT) of ICs have occurred in vertebrates. In addition, the discovery of intact transposases, perfect TIRs and target site duplications of ICS suggests that this family may still be active in Insecta, Arachnida, frogs, and fish.

Conclusions: Exploring the diversity of Tc1/mariner transposons and revealing their evolutionary profiles will help provide a better understanding of the evolution of DNA transposons and their impact on genomic evolution. Here, a newly discovered family (DD36E/Incomer) of Tc1/mariner transposons is described in animals. It displays a similar structural organization and close relationship with the known DD34E/TC1 elements, but has a relatively narrow distribution, indicating that DD36E/IC might have originated from the DD34E/TC1 family. Our data also support the hypothesis of horizontal transfer of $\mathcal{C}$ in vertebrates, even invading one lineage of mammals (bats). This study expands our understanding of the diversity of Tc1/mariner transposons and updates the classification of this superfamily.
\end{abstract}

Keywords: TC1/mariner transposons, DD36E, Horizontal transfer

\footnotetext{
* Correspondence: cysong@yzu.edu.cn

†Yatong Sang and Bo Gao contributed equally to this work.

${ }^{1}$ Institute of Animal Mobilome and Genome, College of Animal Science \&

Technology, Yangzhou University, Yangzhou 225009, Jiangsu, China

Full list of author information is available at the end of the article
}

(c) The Author(s). 2019 Open Access This article is distributed under the terms of the Creative Commons Attribution 4.0 International License (http://creativecommons.org/licenses/by/4.0/), which permits unrestricted use, distribution, and

reproduction in any medium, provided you give appropriate credit to the original author(s) and the source, provide a link to the Creative Commons license, and indicate if changes were made. The Creative Commons Public Domain Dedication waiver (http://creativecommons.org/publicdomain/zero/1.0/) applies to the data made available in this article, unless otherwise stated. 


\section{Background}

Fragments of DNA sequences, which can autonomously replicate and translocate between chromosomes, are called transposable elements (TEs) or transposons. The first transposon was discovered by Barbara McClintock in maize [1]. They were subsequently detected in various organisms, such as bacteria, fungi, and insects. Based on their mechanism of transposition, TEs can be divided into two major classes: class I transposons transpose by RNA (also called retrotransposons); and class II transposons transpose by DNA (also called DNA transposons). Class II transposons can be further divided into three subcategories: the classical "cut-and-paste" DNA transposons, "rolling circle" DNA transposons, and "self-synthesizing" DNA transposons [2]. For a long time, transposons were designated as "junk DNA" in the genome and ignored. However, with the completion of large-scale genome sequencing projects, transposons have been found to exist in almost all genomes. It is now believed that TEs play important roles in genomic evolution and are regarded as important factors in determining genome expansion. They can simultaneously modify gene structures, provide sources of regulatory sequences [3, 4], and have important impacts on the structure and evolution of the genes of eukaryotes $[5,6]$.

Tc1/mariner is an important superfamily of "cut-andpaste" transposons, which was first discovered in Drosophila mauritiana $[7,8]$. Elements in this superfamily are generally $1300-2400$ base pairs (bp) in size and encode a 340-amino acid (aa) transposase that is flanked by TIRs and a TA target site duplication (TSD) at each end [9]. Tc1/mariner transposases contain a DNAbinding domain (DBD), which harbors two helix-turnhelix (HTH) motifs [10], a conserved GRPR-like sequence between the two HTH motifs [11], and a conserved catalytic amino acid triad motif (DDE/D), which usually interacts with a divalent cation $\left(\mathrm{Mg}^{+2}\right.$ or $\left.\mathrm{Mn}^{+2}\right)$ to perform the biochemical steps of the transposition reaction [12]. The distance between the first two " $D$ " amino acids is variable across different transposase families, while the distance between the " $\mathrm{D}$ " and the third "D/E" is highly conserved. Accordingly, the length of this spacer has been used to characterize this transposase family [13]. Regarding variations of the DDE/D signature motif, Tc1/mariner elements have been classified into eight distinct families: DD34E/Tc1, DD34D/mariner, DD37E/TRT, DD37D/maT, DD39D, DD $\times \mathrm{D} /$ pogo, $\mathrm{DD} 41 \mathrm{D}$, and $\mathrm{DD} \times \mathrm{E}$ [14]. Although many Tc1/mariner transposons have been identified in nature, only a few naturally active Tc1/mariner transposons have been discovered, such as Thm3 [15], Tc1 [16], Tc3 [17], and Mos1 [18]. This is because insertion of the transposon results in instability of the genome; therefore, long-term purifying natural selection, genetic drift, and mutations can result in gradual inactivation or even disappearance of transposons within the host genome [19, 20]. In addition, studies have shown that horizontal transfer (HT) of transposons is an important way to avoid inactivation and extinction. The HT of transposons between species is considered to be an important driver of genomic variation and biological innovation [21]. Almost all kinds of eukaryotic superfamily TEs have been proved to be capable of HT [21], while the Tc1/mariner superfamily seems to be more prone to this behavior [22]. More than $1200 \mathrm{HT}$ events of Tc1/mariner have been reported to date [23].

Gaining further insight into the evolutionary profile of Tc1/mariner transposons will provide a better understanding of DNA transposon evolution and their impact on genome evolution. Here, we uncovered a new family of Tc1/mariner transposons, which is closely related to the DD34E/Tc1 family, but forms a distinct clade and harbors a DD36E motif in its DDE domain. We also report the taxonomic distribution of ICs, describe the structural organization of these elements, and provide evidence to support the occurrence of HT among vertebrates, and the invasion of this family in one lineage of mammals (bats).

\section{Results}

\section{Taxonomic distribution of ICs}

Using TBLASTN searching (https://blast.ncbi.nlm.nih. gov/Blast) with the DD34E references (Passport, Prince, Quetzal, and Sleeping Beauty) as queries, we identified an intact Tc1/mariner-like transposon in Rhinella marina, where it harbors a newly identified transposase family with a DD36E motif, which is close to, but distinct from the previously known family of DD34E/Tc1. We named this newly discovered member of the Tc1/mariner superfamily Incomer or DD36E/IC. To investigate the evolutionary profile of this family, a TBLASTN search against all the available organism genomes of prokaryotes (bacteria and archaea) and the eukaryotes (Protozoa, Animalia, Fungi, Plantae, and Chromista) deposited at the National Center for Biotechnology Information (NCBI) database (https://www.ncbi.nlm.nih.gov) was performed using the $I C$ transposase (346 aa) of $R$. marina as the query term. The obtained IC transposases were in turn used as query terms to identify more $I C$ elements. These searches revealed that this family was absent in prokaryotes, and displayed a narrow distribution in eukaryotes with a main distribution in the animal kingdom (Fig. 1 and Additional file 1: Table S1). Further analysis revealed that the $I C$ transposons were present in invertebrate and vertebrate groups, but that $I C$ showed a very narrow distribution in invertebrates, only present in two classes (four species of Insecta and nine species of Arachnida) among the Arthropoda. In vertebrates, IC 


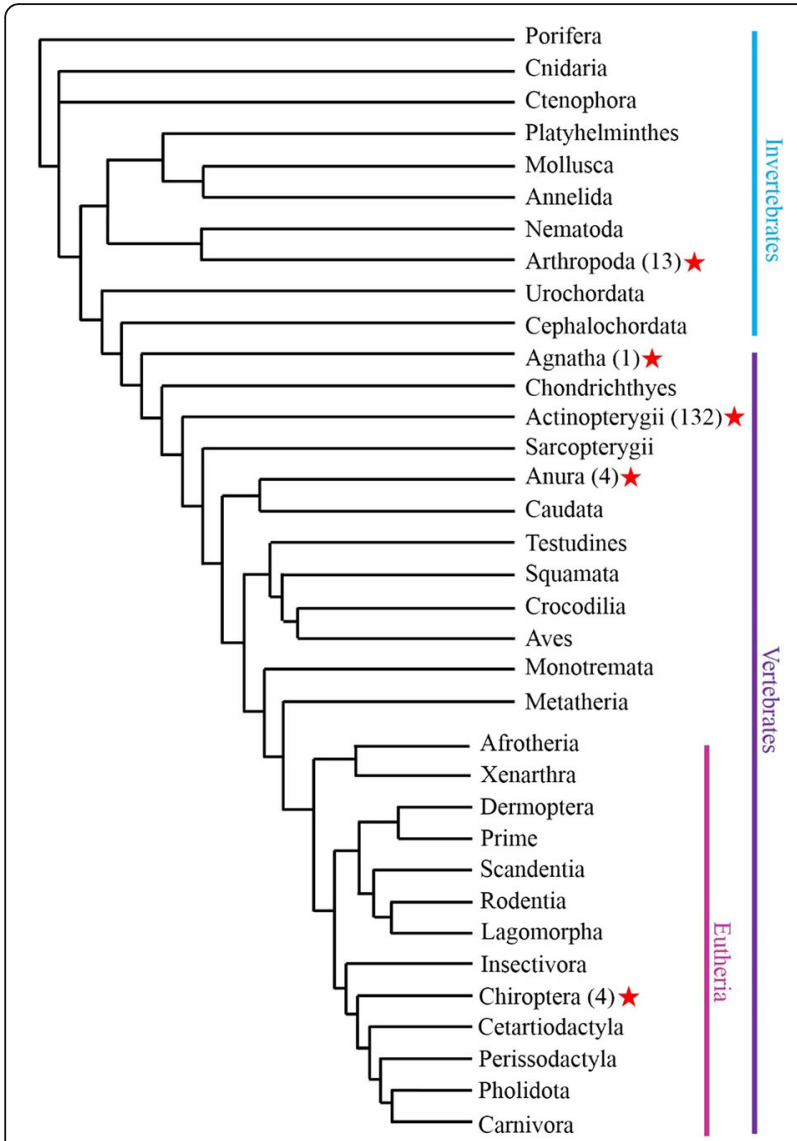

Fig. 1 Taxonomic distribution of IC elements among Eukaryota. IC elements identified in the branches are shown as red stars, and the numbers in parentheses represent the number of species that with IC elements in each branch

transposons were found in 141 species among four classes, including Agnatha (one species of jawless fish), Actinopterygii (132 species of ray-finned fish), Amphibia (four species of frogs), and Mammalia (four species of bats). This family did not undergo significant expansion in most classes of animals, but radiated in the Actinopterygii, where it displays an extensive distribution in 132 species of 38 orders compared with other classes (Additional file 2: Figure S1). In addition, most of the $I C$ transposons are present as truncated copies: thus, in Actinopterygii, more than half of the species (76/132) contain full-length $I C$ elements (with two detectable TIRs), but only 33 species contain intact $I C$ copies (with an intact transposase and two detectable TIRs). Among the Anura, four species contain full-length $I C s$ and three of them harbor intact copies of ICs. Intact IC copies were also detected in one species of Agnatha, while all ICs in the four species (Myotis lucifugus, Eptesicus fuscus, M. davidii, M. brandtii) of the Chiroptera are present as truncated copies and an intact copy was not detectable. Among the Arthropoda, four species of
Insecta and seven species of Arachnida contain fulllength $I C$ copies, with four and three species harboring intact $I C$ elements, respectively (Table 1 ). The discovery of intact transposases, perfect TIRs, and TSDs of ICs suggests that this family could still be active in insects, Arachnida, frogs, and fish.

In addition, the copy number of ICs in the genomes of different organisms varies dramatically, from only one copy ( $>90 \%$ of identity and $>1000$ bp in length) in some organisms (e.g., Seriola dumerili, Amphilophus citrinellus, Gadiculus argenteus, and Xenocatantops brachycerus) to several thousand copies in a few species including R. marina, Esox lucius, and Clitarchus hookeri. $I C s$ have undergone significant amplification in $R$. marina; i.e., more than 5000 copies of $I C s$ were detected and more than half of them are full-length copies (2949 copies). ICs are also enriched in the genomes of $E$. lucius (5418 copies) and C. hookeri (3, 956 copies) (Additional file 1: Table S1). Previous studies revealed that both the $R$. marina and $C$. hookeri genomes contain high repeat contents, with 63.9 and $51.6 \%$ of their genomes covered by repeats, respectively $[24,25]$. These data indicate that some organisms might be more susceptible to HT of transposons and tend to enrich repeated copies in their genomes.

\section{Structural organization of ICs}

The structural organization of ICs was found to be highly conserved across different classes of animals including insects, Arachnida, fish, frogs, and bats. Most intact IC transposons had a total length of about $1.2 \mathrm{~kb}$ and harbored a single open reading frame (ORF) encoding a protein of about 346 aa in length (range 335-382 aa) flanked by short TIRs (22-32 bp) (Table 1 and Fig. 2). The $I C$ elements were found to be flanked by TA target site duplication (Table 1). The intact $I C$ transposon in $R$. marina, representing a typical structure of this family, is 1225 bp long, encoding a 346 aa transposase and flanked by $29 \mathrm{bp}$ TIRs (Fig. 2). Several conserved motifs, including six predicted helices in two HTHs and GRPR in the $\mathrm{N}$-terminal DBD, and a nuclear localization sequence (NLS), which are characteristic of Tc1/mariner transposases [11], were identified in most $I C$ transposases by in silico prediction. The DDE signature and its spacing (36 aa) in the DDE domain seems to be highly conserved across the $I C$ family (Fig. 2 and Additional file 2: Figure S2). All ICs in the four genomes of bats (M. lucifugus, $E$. fuscus, $M$. davidii, and $M$. brandtii) presented as truncated copies; the longest ICs in these species are 1220, 1487, 1056, and $1005 \mathrm{bp}$, respectively, and encode a truncated transposase (235 aa) containing a partial DBD and a DDE domain. Moreover, the TIR lengths of bat ICs also vary slightly in three bat species compared with the IC TIRs in the genomes of other organisms, with 
Table 1 Taxonomic distribution of IC elements

\begin{tabular}{|c|c|c|c|c|c|c|}
\hline Taxonomic distribution & Mammalia (Bat) & Amphibia (Frog) & Actinopterygii & Myxini & Arachnida & Insecta \\
\hline Number of species containing IC & 4 & 4 & 132 & 1 & 9 & 4 \\
\hline Number of species containing full IC & 4 & 4 & 76 & 1 & 7 & 4 \\
\hline Number of Species containing intact IC & 0 & 3 & 33 & 1 & 3 & 3 \\
\hline Length of full IC & $1005-1487$ & $1194-1225$ & $1099-1467$ & 1226 & $1200-1234$ & $1213-1226$ \\
\hline Length of intact IC & $\mathrm{N}$ & $1194-1225$ & $1169-1229$ & 1226 & $1220-1224$ & $1217-1226$ \\
\hline Transposase length of intact IC & $\mathrm{N}$ & 346 & $314-356$ & 346 & $346-382$ & $346-366$ \\
\hline TIR length of intact IC & $\mathrm{N}$ & $28-29$ & $24-32$ & 28 & $25-27$ & $25-28$ \\
\hline TSD & TA & TA & TA & TA & TA & TA \\
\hline
\end{tabular}

$126 \mathrm{bp}$ in $M$. lucifugus, $51 \mathrm{bp}$ in $M$. davidii, and $55 \mathrm{bp}$ in M. brandtii (Fig. 2). We also found a high copy number of miniature inverted-repeat transposable elements (MITEs) derived from ICs in the four bat genomes and most of these have a length of about $810 \mathrm{bp}$. Some MITE copies also encode the truncated transposase (235 aa) (Fig. 2 and Table 2).

\section{Phylogenetic analysis and evidence for multiple HT events of ICS}

To accurately establish the evolutionary relationships of the $I C$ elements that we identified, the conserved DDE domain of the identified $I C$ transposases were aligned to the 28 known DNA transposases representing the eight families in the Tc1/mariner superfamily based on MAFFT v 7.310 [26]. The alignment was used for phylogenetic analysis using the maximum-likelihood method implemented in IQ-TREE [27], and the TP36_RB, which is an insertion element family identified in bacteria, and close to the Tc1/mariner transposases [28] was used as the outgroup. The polygenetic tree confirmed that all these elements identified belong to the DD36E/IC family, which is more closely related to the DD34E/Tc1 family than to other families of Tc1/mariner (e.g., DD34D/ mariner and $\mathrm{DD} \times \mathrm{D} /$ pogo; Fig. 3). The deduction is also well supported by the highest transposase sequence identity between these two families (Fig. 4), and further confirmed by the phylogenetic tree generated by using the alignment of the full-length transposases (Additional file 2: Figure S3).

The above phylogeny showed that IC elements identified in this study could be classified into four major clusters: Cluster A includes five species (one frog and four bats); Cluster B includes 59 species (three frogs and 56 fishes); Cluster $\mathrm{C}$ includes five species (two fishes and three insects); and Cluster D includes four species (all insects; Additional file 2: Figure S4). Phylogenetic analysis also suggested that the host and IC phylogenies were incongruent (Fig. 3 and Additional file 2: Figure S5), which implied that $I C$ elements might have been exposed to several episodes of HT. To test this, pairwise distances between recombination-activating gene 1 (RAG1) and all consensus sequences of ICs in vertebrate were calculated and compared, which are usually used to infer the HT events of transposons in vertebrates [29, 30]. As illustrated in Fig. 5, for almost all (177/196) pairwise comparisons, the distances computed for IC (average 0.121; standard deviation, $\mathrm{SD} \pm 0.067$; range $0.001-$ $0.259)$ are much lower than those calculated for RAG1 (average 0.278; $\mathrm{SD} \pm 0.106$; range 0.009-0.457) (Additional files 3: Table S2). TEs are known to evolve neutrally after insertion in a host genome [31]; thus, TE distances between taxa are expected to be higher than distances between orthologous genes and to evolve faster than the host genes that evolve under purifying selection because of functional constraints under vertical transmission of the TEs. The low pairwise $I C$ distances, combining deep divergence times, with most species involved sharing a last common ancestor more than 110 million years ago (Ma), indicate multiple HT events of ICs in vertebrates.

To further illustrate the HT profiles of $I C$ elements in animal, we compared the average sequence identities of IC elements across species and clusters, which was summarized in Fig. 6. And the sequence identity matrix showed that most $I C$ s in cluster B represent higher sequence identities ( $>78 \%$; average $87.96 \pm 5.41 \%$ ) between species, indicating that the cluster $\mathrm{B}$ may represent a very young $\mathrm{HT}$ event, while high and low sequence identities of ICs between species co-exist in cluster A, C, and $\mathrm{D}$, indicating that these cluster may experience young and old invasions of ICs.

\section{Discussion}

Expanding the diversity of the $T c 1 /$ mariner superfamily Compared with other DNA transposons, the Tc1/mariner superfamily might not only be the most widely distributed group of transposons in nature, but also displays the highest diversity. Phylogenetic analyses based on the distinct "DDE/D" signatures of transposases from diverse organisms suggest that Tc1/mariner transposons comprise at least eight families in 

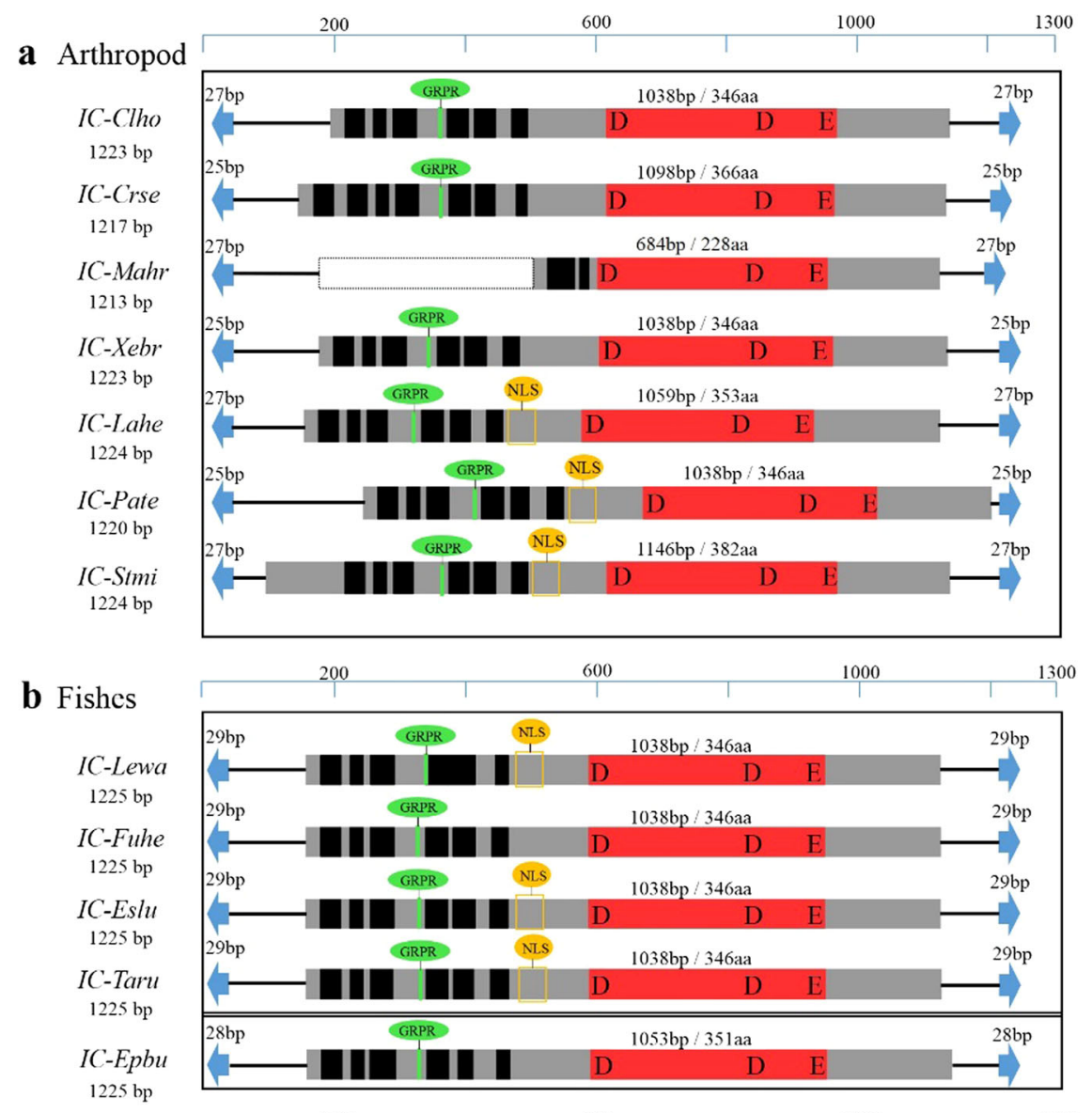

c Frogs

IC-Rhma

$1225 \mathrm{bp}$

IC-Napa

$1194 \mathrm{bp}$

IC-Xetr

$1225 \mathrm{bp}$

IC-Raca

1212 bp

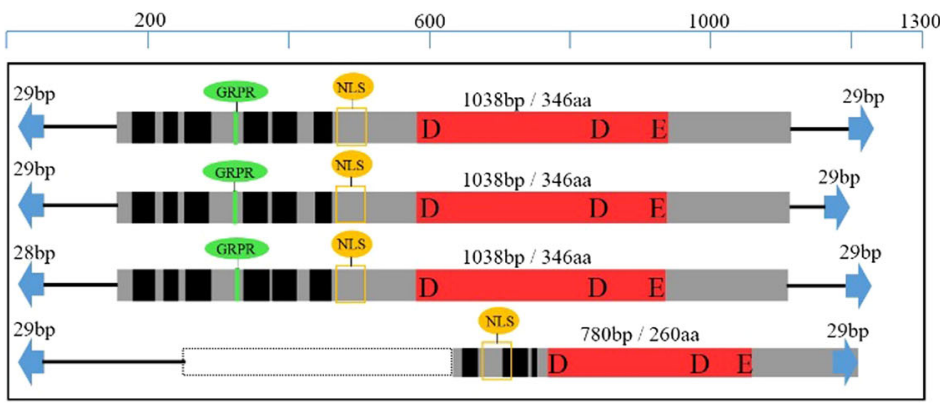

d Bats

$I C-M y l u$
$1220 \mathrm{bp}$
$I C-E p f u$
$1487 \mathrm{bp}$
$I C-M y d a$
$1056 \mathrm{bp}$
$I C-M y b r$
$1005 \mathrm{bp}$
$I C-M I T E$
$810 \mathrm{bp}$

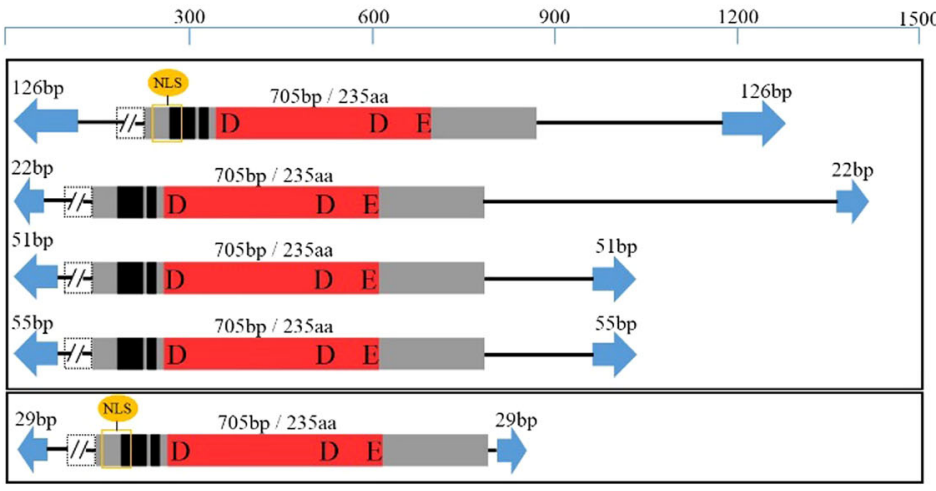

Fig. 2 (See legend on next page.) 
(See figure on previous page.)

Fig. 2 Structural organization of ICS. The blue arrows represent TIRs; the black rectangles represent HTH motifs; green rectangles represents GRPR sequences; the orange wire frame represents the NLS; the red rectangles represents catalytic domains, D:aspartic acid, E:glutamic acid; and the gray region represents transposases. The dotted box represents the portion of the transposases that can be deleted in the particular species. Clho:Clitarchus hookeri; Crse:Cryptotermes secundus;Mahr: Machilis hrabei; Xebr:Xenocatantops brachycerus; Lahe:Latrodectus hesperus; Pate:Parasteatoda tepidariorum; Stmi:Stegodyphus mimosarum; Lewa:Leuciscus waleckii; Fuhe:Fundulus heteroclitus; Eslu:Esox lucius; Taru:Takifugu rubripes; Epbu:Eptatretus burgeri; Rhma:Rhinella marina; Napa:Nanorana parkeri; Raca:Rana catesbeiana; Xetr:Xenopus tropicalis. Mylu: Myotis lucifugus;Epfu: Eptesicus fuscus; Myda:Myotis davidii; Mybr:Myotis brandtii

eukaryotes: (1) DD34D/mariner; (2) DD39D; (3) DD37E; (4) DD34E/Tc1; (5) DD37D; (6) DD35E; (7) $\mathrm{DD} \times \mathrm{D} /$ pogo; and (8) DD41D [14, 32]. Here, we expanded the diversity of the Tc1/mariner superfamily and identified the ninth family (DD36E/IC) of these transposons, discontinuously distributed in 13 species of invertebrates and 141 species of vertebrates. The structure organizations of ICs are very similar to the known DD34E/Tc1 elements and present all the hallmark features of Tc1-like elements, including the existence of a transposase of about 346 aa in length, a DDE motif, two HTH motifs in the DNA-binding domains, TIRs, and a TSD TA at each end. However, the spacing of the DDE motif within the catalytic domain of $I C$ is unique, with 36 aa (DD36E) separating the second aspartic acid and the glutamic acid residues. The phylogenetic analyses place $I C$ elements in a distinct group separate from other known families of Tc1/mariner transposons, which suggests that these elements constitute a newly discovered family within the Tc1/mariner superfamily. Furthermore, the increasing numbers of newly discovered families of the Tc1/mariner superfamily as more genome sequencing data becomes available $[29,33]$ indicates that the diversity of Tc1/mariner superfamily may be far greater than currently known.

\section{Origin of ICs and relationship to the DD34E /Tc1 family}

The host of the earliest branching IC clade was the phylum Arthropoda, the next earliest branching clade included elements from Agnatha. However, here the phylogenetic trees generated by using both of the fulllength transposases and the DDE domains indicated that the IC family is closest to the DD34E/Tc1 family; thus, an origin in the DD34E/Tc1 family for the entire group of $I C s$ in metazoans is more plausible, given that several DD34E/Tc1 elements, such as Minos [34], Bari [35], S element [36], Quetzal [37], and Topi [38], were also identified in Arthropoda. To date, diverse DD34E/Tc1 elements have been identified and described [34-40]; the DD34E/Tc1 family seems to display a more extensive distribution than DD36E/IC and can be classified into several subfamilies, such as Passport-like, Frog Princelike, SB-like, Bari-like, Minos-like [24], and Gambol [41], although the intra-group classification of DD34E/Tc1 is still ambiguous. The structural organization of $I C s$ is very similar to some DD34E/Tc1 elements identified in the neoteleost genomes of Actinopterygii [32], Bari (Drosophila melanogaster) [35], and Topi (Anopheles gambiae) [38] identified in Arthropoda, where these families are relatively shorter in total length (about 1200 bp) and contain a single ORF (about 340 aa) flanked by short TIRs (about $30 \mathrm{bp}$ ). The structural organization of transposases, including the protein motifs of six helices, GRPR, and NLS in the DBD domain, between ICs and DD34E/Tc1 is very similar as well [32, 35, 38]. Furthermore, our data also indicated that the $I C$ family and DD34E/Tc1 have the highest transposase sequence identity compared to other families. Taken together, these data indicate that the DD36E/IC might originate from the DD34E/Tc1 family.

Table 2 Incomer in bats

\begin{tabular}{|c|c|c|c|c|c|}
\hline Species & TE name & Length & Transposase length & Copy number & $\begin{array}{l}\% \text { Ave. Divergence } \\
\pm \text { SE }\end{array}$ \\
\hline \multirow[t]{2}{*}{ M. lucifugus } & IC-MITE1-Mylu & 1220 & 235 & 407 & $4.4 \pm 0.08$ \\
\hline & IC-MITE2-Mylu & 810 & 235 & 951 & $5.2 \pm 0.09$ \\
\hline \multirow[t]{2}{*}{ E. fuscus } & IC-MITE1-Epfu & 1487 & 235 & 1 & $N A^{a}$ \\
\hline & IC-MITE2-Epfu & 810 & 235 & 367 & $3.9 \pm 0.05$ \\
\hline \multirow[t]{2}{*}{ M. davidii } & IC-MITE1-Myda & 1056 & 235 & 4 & NA \\
\hline & IC-MITE2-Myda & 810 & 235 & 147 & $5.3 \pm 0.6$ \\
\hline \multirow[t]{2}{*}{ M. brandtii } & IC-MITE1-Mybr & 1005 & 235 & 48 & $4.0 \pm 0.15$ \\
\hline & IC-MITE2-Mybr & 810 & 235 & 215 & $3.5 \pm 0.06$ \\
\hline
\end{tabular}

SE standard error

${ }^{a}$ Average percent divergence could not be determined for full-length $I C$ elements due to low copy number 


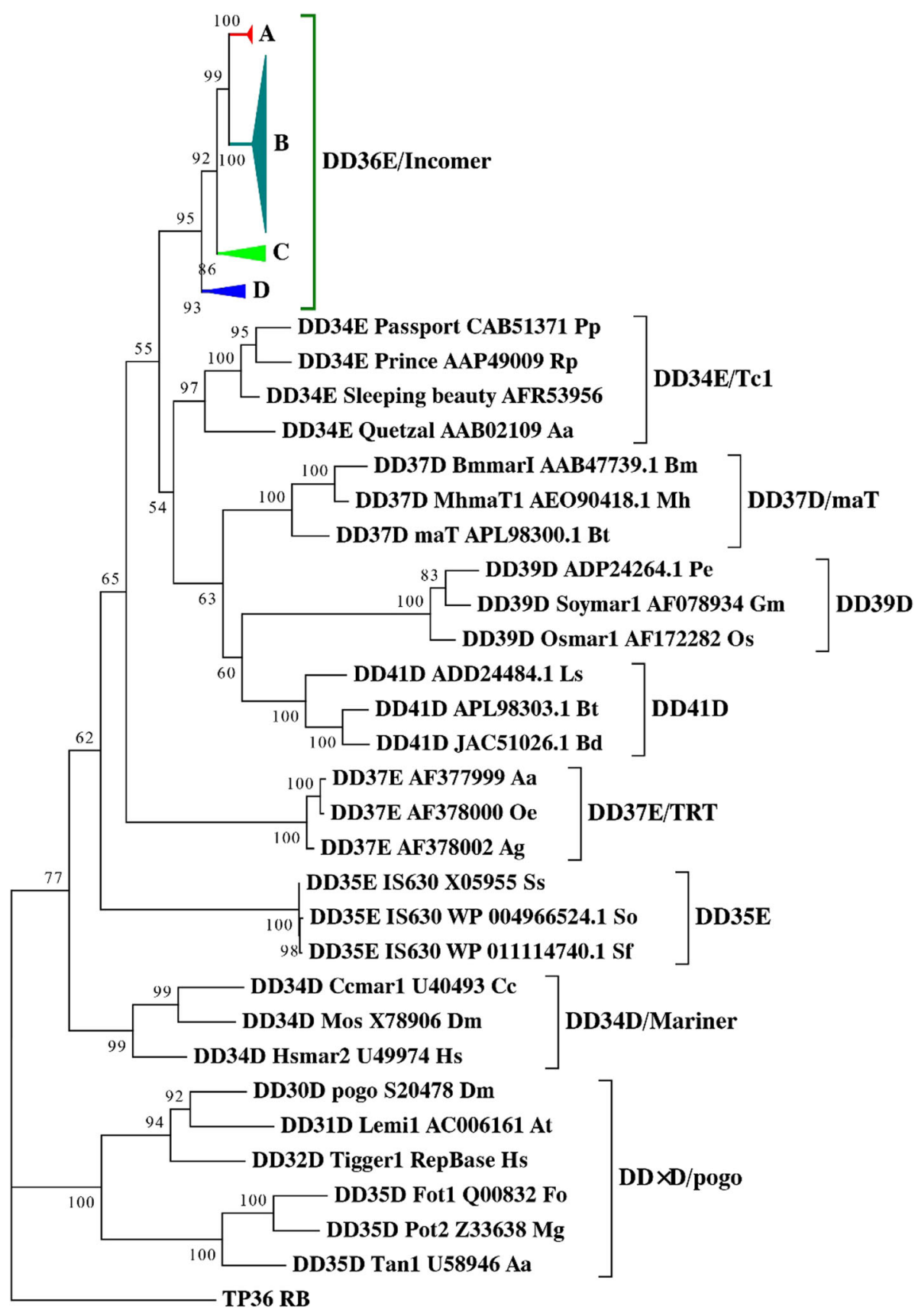

Fig. 3 Phylogenetic tree of IC elements identified in this study with eight other members of the TC1/mariner superfamily based on their transposases. Bootstrapped (1000 replicates) phylogenetic trees were inferred by using the Maximum Likelihood method in IQ-TREE [27]. Each sequence (except the DD37E and DD41D subclasses) contains the name of the transposon, the gene sequence number corresponding to the transposon, and the Latin abbreviation of the species in which the transposon is located. Pp:Pleuronectes platessa;Rp:Rana pipiens; Aa:Anopheles albimanus; Bm:Bombyx mori; Mh:Misgolas hubbardi; Bt:Bactrocera tryoni; Pe:Phyllostachys edulis; Os:Oryza sativa; Gm:Glycine max; Ls:Lepeophtheirus salmonis; Bd:Bactrocera dorsalis; Aa:Aedes atropalpus; Oe:Ochlerotatus epactius; Ag:Anopheles gambiae; Serratia odorifera Sf:Shigella flexneri; Ss:Shigella sonnei; Hs:Homo sapiens; Cc:Ceratitis capitata; Dm:Drosophila mauritiana; Dm:Drosophila melanogaster; At:Arabidopsis thaliana; Aa:Aspergillus awamori; Fo:Fusarium oxysporum; Mg:Magnaporthe grisea

Distribution and activity of ICs in animals Compared with the DD34E/Tc1 family, which displays extensive expansion in invertebrates and vertebrates [32, $41,42]$, even among fungi [43], ICs show a very relatively narrow distribution in invertebrates and are only present in 13 species of two classes (Insecta and Arachnida) of Arthropoda. In vertebrates, ICs were detected in 141 species of four classes (Agnatha, Actinopterygii, 


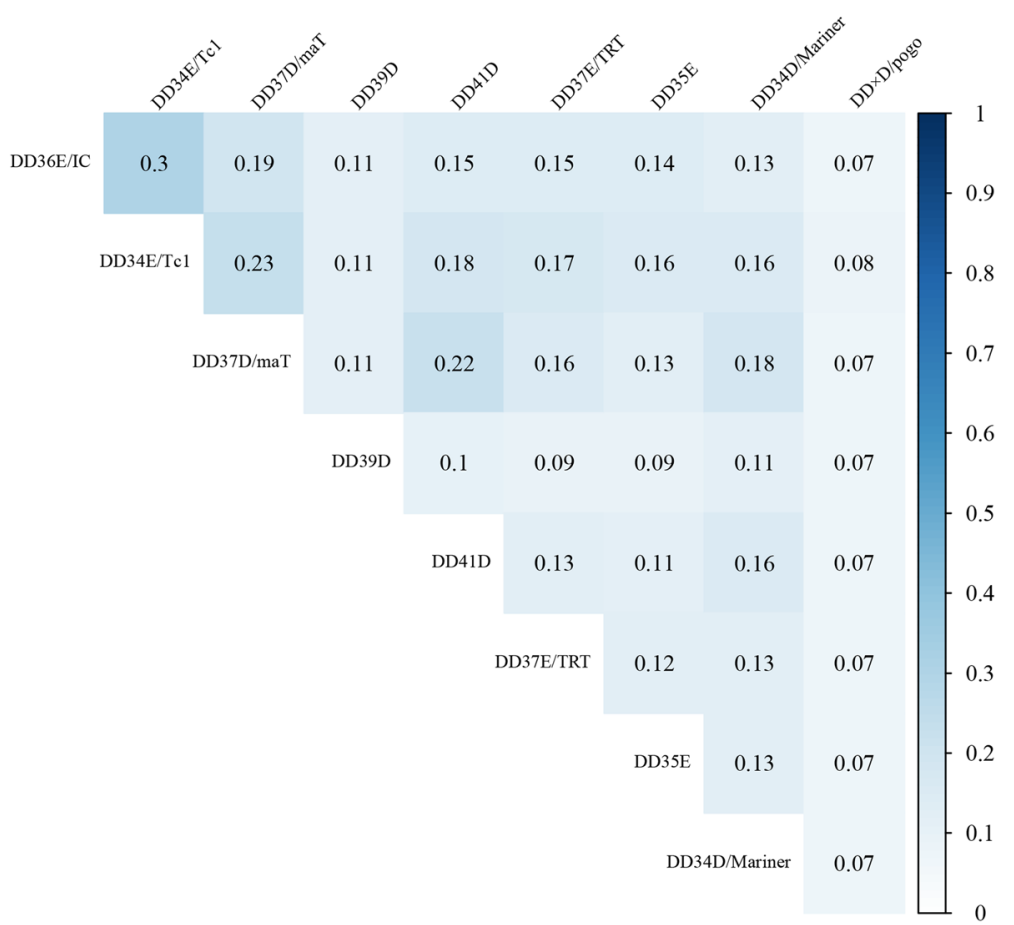

Fig. 4 Sequence identities between $/ C$ family and eight other families. The sequence identities were measured by pairwise comparisons of full-length transposases

Amphibia, and Mammalia). ICs display a slight burst in the Actinopterygii with an expansion into 132 species of 38 orders and have even invaded the mammalian lineage (Chiroptera) lineage, that has been suggested to be more susceptible to HT of transposons than other groups, and have experienced HT events of most DNA transposons (hATs, piggyBacs, Tc1/mariner, and Helitron) [44]. Here we provide evidence to support another HT event from a newly discovered family of Tc1/mariner transposons in bats, suggesting that some DNA transposons tend to show recurrent invasion of some mammalian lineages. This was confirmed in the evolution of the $h A T$ superfamily, which was also found to undergo repeated HT events in mammals [30]. These data also indicate that HT of DNA transposons has contributed significantly to shaping and diversifying the genomes of multiple mammalian species, although HT of DNA transposons is relatively rare in mammals. In addition, the taxonomic distribution of ICs revealed by this study might have been underestimated, because of inefficient sequencing

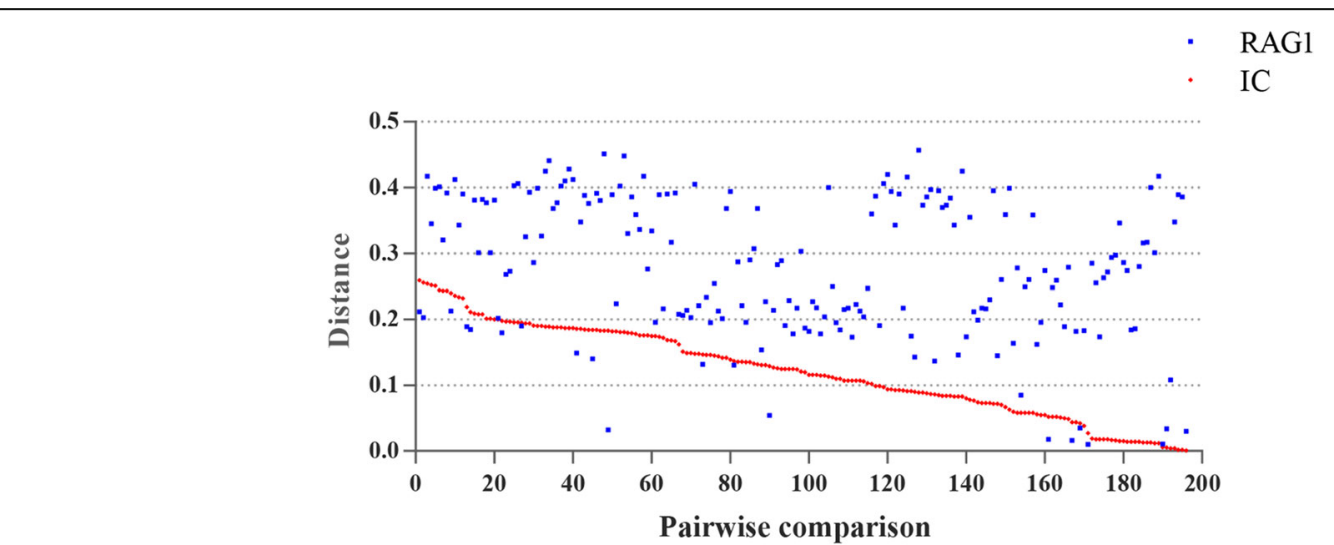

Fig. 5 Pairwise distances of IC elements and RAG1. The distances are obtained from all possible pairwise comparisons ( $n=196$; labeled on the $x$ axis) between the four (Cluster A) and 20 (Cluster B) species in which ICs were identified and complete. The coding sequence (CDS) regions of the RAG1 gene in the NCBI database are available (Additional file 7: Text S3 and Additional file 8: Text S4) 


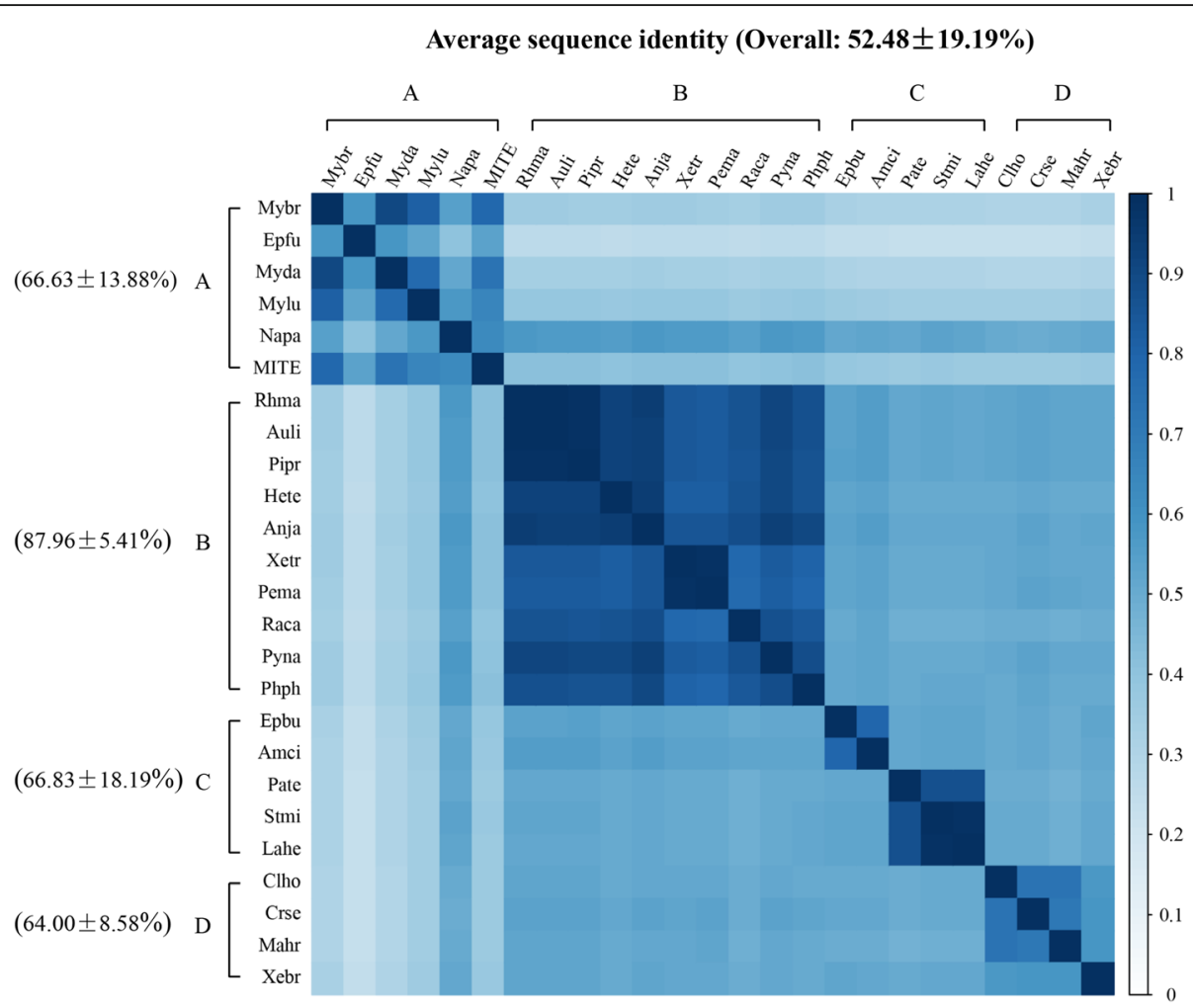

Fig. 6 Sequence identities between $/ C$ elements among species. The sequence identities were measured by pairwise comparisons of full-length IC consensus sequences. Auli: Austrofundulus limnaeus; Pipr: Pimephales promelas; Hete: Helostoma temminkii; Anja: Anguilla japonica; Pema: Periophthalmus magnuspinnatus; Pyna: Pygocentrus nattereri; Phph: Phycis phycis

or assembly technologies, or the unavailability of some genome sequences.

Several families of Tc1/mariner have been suggested to be active (e.g., Tc1, TRT, Tana1, and pogo) [15, 29, $32,39,45]$ and some of them have been shown to transpose experimentally, such as Passport and Thm3 in fish $[15,39]$. Here, intact copies of ICs were identified in 33 species of bony fish, three species of frogs, one species of jawless fishes, and seven species of Arthropoda. They did not harbor internal stop codons or frameshift mutations and presented all expected functional domains, as well as intact TIRs, combining the narrow taxonomic distribution of this family, suggesting that $I C$ is young and might be associated with current or recent activity in these host genomes.

\section{Conclusions}

Our results represent the first in silico evidence for a newly identified family (DD36E/IC) of the Tc1/mariner superfamily and uncover the evolutionary landscape of this family in nature. This family is about $1200 \mathrm{bp}$ in total length, encoding a transposase of $\sim 346$ aa flanked by short TIRs (about $30 \mathrm{bp}$ ), and mainly distributed in vertebrates (141 species), with a restricted presence in invertebrates (13 species). This family can be subdivided into four distinct clusters based on the catalytic domain signature DD36E. Based on structural organization, protein motifs and phylogenetic analyses, the $I C$ transposons are closely related to DD34E/Tc1 elements, indicating a recent common ancestor. Furthermore, evidence for HT events in vertebrates is well supported for this family. We have also demonstrated the presence of $I C$ in the bat lineage of mammals. We propose an update of the classification of the Tc1/mariner superfamily and illustrate the evolutionary relationships among these distinct families.

\section{Methods}

\section{Identification and copy number determination of IC}

The IC family was first identified in $R$. marina by TBLASTN searching with the DD34E references (Passport, Prince, Quetzal, and Sleeping beauty), then its taxonomic distribution was investigated by a TBLASTN search against all the available organism genomes deposited at the NCBI database using the IC R. marina transposase (346 aa) as query. The IC transposon was considered to be present in one species when a unique DD36E motif of the transposon catalytic domain was detected. The obtained $I C$ transposases were in turn used as queries to identify more $I C$ elements. To determine 
the boundaries of these elements in each species, the best hits were extracted with a $2 \mathrm{~kb}$ flanking sequences, aligned using the ClustalW program within the BioEdit tool [46], and the transposon boundaries were then checked manually. In addition, copies $(>10)$ in each species were also aligned using the ClustalW program within the BioEdit tool [46] and their consensus sequences were reconstructed using the above multiple alignments in each genome using DAMBE after gaps were removed [47]. If one genome sequence contained a low copy number $(<10)$, the best hit was used as the representative sequence of $I C$ in this species. Then, these consensus sequences were entered into BLASTN for each host genome to estimate copy numbers. All BLAST hits with more than $1000 \mathrm{bp}$ in size and $90 \%$ identity were used to calculate copy numbers. The copy of the transposon possessing the complete TIR sequence and encoding the entire or longest transposase was used as a representative sequence for the transposon in the species for further structural organization and polygenetic analysis.

\section{Sequence analyses}

TIRs were manually checked by using the BioEdit tool [46]. The potential ORF of Incomer used in the present study was predicted by Genscan (http://hollywood.mit. edu/GENSCAN.html). Protein secondary structures of $I C$-encoded transposases were predicted using PSIPRED [48]. Putative NLS motifs were predicted using PSORT II Prediction as provided in the PSORT Internet server (http://psort.nibb.ac.jp/). Multiple alignments of these elements were created by MUSCLE [49]. Shading and minor manual refinements of these aligned sequences were deduced using GeneDoc [50]. The pairwise divergence between elements and the average divergence from the consensus sequence were calculated using Kimura's 2-parameter method in MEGA software v. 7.2.06 [51]. Sequence identities between $I C$ family and eight other families were measured with the pairwise comparisons of full-length transposases by using the BioEdit tool [46]. And sequence identities between IC elements among species were measured by pairwise comparisons of full-length $I C$ consensus sequences, and $I C s$ in 10 species in cluster B were selected as the representatives for this analysis.

\section{Phylogenetic and $\mathrm{HT}$ analyses}

The conserved DDE domain of the identified IC transposases and full-length transposases were aligned to the 28 known DNA transposases representing eight families from Tc1/mariner superfamily separately by MAFFT v 7.310 [26] (Additional file 4: Text S1 and Additional file 5: Text S2). The species that only had highly fragmented copies and incomplete DD36E motifs in their genome were not included in this analysis. Transposase sequences of DD34E/Tc1, DD34D/Mariner, DD37D/maT, DD39D, DD41D, $\mathrm{DD} 35 \mathrm{E}, \mathrm{DD} \times \mathrm{D} /$ pogo and DD37E/TRT were downloaded from GenBank. The best-suited aa substitution model for these data was the VT + G4 model according to BIC which were selected by ModelFinder embed in IQ-TREE program [27, 52]. Bootstrapped (1000 replicates) phylogenetic trees were inferred by using the maximum-likelihood method in IQ-TREE (v. 1.6.1) [27] .

Coding sequence of RAGlgenes were used in the comparison with transposon distance, with the purpose of testing HT hypothesis. Their accession numbers were listed in (Additional file 6: Table S3). Species that cannot find the complete CDS region of the RAG1 gene in NCBI database are not included in this calculation. Multiple alignments of RAG1 and $I C$ were created using MUSCLE [49]. Then, comparison distances of RAG1 and IC were calculated using MEGA software v. 7.2.06 [51] (pairwise deletion, maximum composite likelihood) based on two aligned files (Additional file 7: Text S3 and Additional file 8: Text S4).

\section{Supplementary information}

Supplementary information accompanies this paper at https://doi.org/10. 1186/s13100-019-0188-x.

Additional file 1: Table S1. Taxonomic distribution of Incomer.

Additional file 2: Figure S1-5. Figure S1: Taxonomic distribution of $/ C$ elements in Actinopterygii. FigureS2: Alignment of domains of Incomer and DD34E/TC1 transposases. FigureS3: Full tree of IC elements with eight other members of the Tc1/mariner superfamily based on their fulllength transposases FigureS4: Full tree of $\mathcal{I C}$ elements with eight other members of the TCl/mariner superfamily based on their DDE/D motifs. FigureS5: Time tree of species harboring $/ C$ elements.

Additional file 3: Table S2. Distance of IC elements and RAG1 genes and divergence time of each species using pairwise comparisons. (XLS $52 \mathrm{~kb})$

Additional file 4: Text S1. Sequence alignment of transposase DDE/D domains used to calculate the tree.

Additional file 5: Text S2. Sequence alignment of full-length transposase used to calculate the tree.

Additional file 6: Table S3. Access number of RAG1 genes. Species only with the complete CDS region of the RAG1 gene in the NCBI database are listed.

Additional file 7: Text S3. Alignment of CDS regions of RAG1 genes. Additional file 8: Text S4. Alignment of $I C$ elements.

\section{Abbreviations}

bp: base pairs; DBD: DNA-binding domain; HTH: Helix-turn-helix; Ma: Million years ago; MITEs: Miniature inverted-repeat transposable elements; NLS: Nuclear localization sequence; RAG1: Recombination-activating gene 1; TEs: Transposable elements; TIRs: Terminal inverted repeats 


\section{Acknowledgments}

We thank the Priority Academic Program Development of Jiangsu Higher Education Institutions for their advice on Tc1/mariner database and classification, and computing resources.

\section{Author's contributions}

CS and ZI conceived the study; BG participated in its design. BG, YS, MD, WZ, CC, DS, SW, YW performed the analysis. CS and BG wrote the manuscript. All authors have read and approved the final manuscript.

\section{Funding}

This work was supported by a grant from the Major Projects of National Genetically Modified Organism Breeding (2018ZX08010-08B) and the Natural Science Foundation of China (31671313), the Priority Academic Program Development of Jiangsu Higher Education Institutions and the High-end Talent Support Program of Yangzhou University.

\section{Availability of data and materials}

All data generated or analysed during this study are included in this published article and its supplementary information files.

\section{Ethics approval and consent to participate}

Not applicable.

\section{Consent for publication}

Not applicable.

\section{Competing interests}

The authors declare that they have no competing interests.

\section{Author details}

${ }^{1}$ Institute of Animal Mobilome and Genome, College of Animal Science \& Technology, Yangzhou University, Yangzhou 225009, Jiangsu, China. ${ }^{2}$ Division of Medical Biotechnology, Paul Ehrlich Institute, 63225 Langen, Germany.

Received: 9 September 2019 Accepted: 11 November 2019

Published online: 23 November 2019

\section{References}

1. Mcclintock B. The origin and behavior of mutable loci in maize. Proc Natl Acad Sci U S A. 1950;36:344-55.

2. Feschotte C, Pritham EJ. DNA transposons and the evolution of eukaryotic genomes. Annu Rev Genet. 2007;41:331-68.

3. Li Y, Li C, Xia J, Jin Y. Domestication of transposable elements into microrna genes in plants. PLoS One. 2011;6.

4. Rebollo R, Romanish MT, Mager DL. Transposable elements: an abundant and natural source of regulatory sequences for host genes. Annu Rev Genet. 2012:46:21-42.

5. Almeida LM, Silva IT, Silva WA, Castro JP, Riggs PK, Carareto CM, et al. The contribution of transposable elements to Bos taurus gene structure. Gene. 2007;390:180-9.

6. Shapiro JA. Mobile DNA and evolution in the 21st century. Mob DNA. 2010; $1 \cdot 1-14$

7. Haymer DS, Marsh JL. Germ line and somatic instability of a white mutation in Drosophila mauritiana due to a transposable genetic element. Dev Genet. 1985;6:281-91

8. Jacobson JW, Medhora MM, Hartl DL. Molecular structure of a somatically unstable transposable element in Drosophila. Proc Natl Acad Sci. 1986;83: 8684-8.

9. Lohe AR, Sullivan DT, Hartl DL. Subunit interactions in the mariner tranposase. Genetics. 1996;144:1087-95.

10. Pietrokovski S, Henikoff S. A helix-turn-helix DNA-binding motif predicted for transposases of DNA transposons. Mol Gen Genet. 1997:254:689-95.

11. Plasterk RHA, Izsvák Z, Ivics Z. Resident aliens the Tc1/mariner superfamily of transposable elements. Trends in Genetics. 1999:527-38.

12. Baker TA, Luo L. Identification of residues in the mu transposase essential for catalysis. Proc Natl Acad Sci. 1994;91:6654-8.

13. Yuan Y-W, Wessler SR. The catalytic domain of all eukaryotic cut-and-paste transposase superfamilies. Proc Natl Acad Sci. 2011;108:7884-9.

14. Bouuaert CC, Tellier M. Chalmers R. Microbiology Spectrum: Mariner and the ITm Superfamily of Transposons; 2015.
15. Guo X, Zhang Q, Sun Y, Jiang X, Zou S. Tc1-like Transposase Thm3 of Silver Carp (Hypophthalmichthys molitrix) Can Mediate Gene Transposition in the Genome of Blunt Snout Bream (Megalobrama amblycephala). G3. 2015;5: 2601-10.

16. Emmons SW, Yesner L, Ruan KS, Katzenberg D. Evidence for a transposon in caenorhabditis elegans. Cell. 1983:32:55-65.

17. Collins J, Forbes E, Anderson P. The Tc3 family of transposable genetic elements in Caenorhabditis elegans. Genetics. 1989;121:47-55.

18. Medhora M, Maruyama K, Hartl DL. Molecular and functional analysis of the mariner mutator element Mos1 in Drosophila. Genetics. 1991:128:311-8.

19. Hartl DL, Lohe AR, Lozovskaya ER. Modern thoughts on an ancyent marinere: function, evolution, regulation. Annu Rev Genet. 1997;31:337-58.

20. Robertson HM. Evolution of DNA Transposons in Eukaryotes; 2002. p. 1093110.

21. Sarah S, Clément G, Cédric F. Promiscuous DNA: horizontal transfer of transposable elements and why it matters for eukaryotic evolution. Trends Ecol Evol. 2010:25:537-46.

22. Dupeyron M, Singh KS, Bass C, Hayward A. Evolution of Mutator transposable elements across eukaryotic diversity. Mobile DNA Mobile DNA. 2019;10.

23. Dotto BR, Carvalho EL, Silva AF, Duarte Silva LF, Pinto PM, Ortiz MF, et al. HTT-DB: horizontally transferred transposable elements database. Bioinformatics. 2015:31:2915-7.

24. Wu C, Twort VG, Crowhurst RN, Newcomb RD, Buckley TR. Assembling large genomes: analysis of the stick insect (Clitarchus hookeri) genome reveals a high repeat content and sex-biased genes associated with reproduction. BMC Genomics. 2017.

25. Edwards RJ, Tuipulotu DE, Amos TG, O'Meally D, Richardson MF, Russell TL, et al. Draft genome assembly of the invasive cane toad, Rhinella marina. GigaScience. 2018.

26. Yamada KD, Tomii K, Katoh K. Application of the MAFFT sequence alignment program to large data - reexamination of the usefulness of chained guide trees. Bioinformatics. 2016;32:3246-51.

27. Nguyen LT, Schmidt HA, Von Haeseler A, Minh BQ. IQ-TREE: a fast and effective stochastic algorithm for estimating maximum-likelihood phylogenies. Mol Biol Evol. 2015;32:268-74.

28. Bao W, Jurka MG, Kapitonov W, Jurka J. New superfamilies of eukaryotic DNA tyransposons and their internal divisions. Mol Biol Evol. 2009:26:98393.

29. Zhang HH, Li GY, Xiong XM, Han MJ, Zhang XG, Dai FY. TRT, a vertebrate and protozoan tc1-like transposon: current activity and horizontal transfer. Genome Biology and Evolution. 2016.

30. Gilbert C, Hernandez SS, Flores-Benabib J, Smith EN, Feschotte C. Rampant horizontal transfer of SPIN transposons in squamate reptiles. Mol Biol Evol. 2012:29:503-15.

31. Lampe DJ, Witherspoon DJ, Soto-Adames FN, Robertson HM. Recent horizontal transfer of mellifera subfamily mariner transposons into insect lineages representing four different orders shows that selection acts only during horizontal transfer. Molecular Biology and Evolution. 2003.

32. Gao B, Chen W, Shen D, Wang S, Chen C, Zhang L, et al. Characterization of autonomous families of Tc1/mariner transposons in neoteleost genomes. Mar Genomics. 2017;34:67-77.

33. Zhang HH, Shen $Y H$, Xiong XM, Han MJ, Zhang XG. Identification and evolutionary history of the DD41D transposons in insects. Genes and Genomics. 2016;38:109-17.

34. Metaxakis A, Oehler S, Klinakis A, Savakis C. Minos as a genetic and genomic tool in Drosophila melanogaster. Genetics. 2005.

35. Caizzi R, Caggese C, Pimpinelli S. Bari-1, a new transposon-like family in Drosophila melanogaster with a unique heterochromatic organization. Genetics. 1993.

36. Merriman PJ, Grimes CD, Ambroziak J, Hackett DA, Skinner P, Simmons MJ. $S$ elements: a family of Tc1-like transposons in the genome of Drosophila melanogaster. Genetics. 1995.

37. Ke Z. Quetzal: a transposon of the Tc1 family in the mosquito anopheles albimanus. Genetica. 1996

38. Subramanian RA, Akala OO, Adejinmi JO, O'Brochta DA. Topi, an IS630/Tc1/ mariner-type transposable element in the African malaria mosquito Anopheles gambiae. Gene. 2008.

39. Clark KJ, Carlson DF, Leaver MJ, Foster LK, Fahrenkrug SC. Passport, a native Tc1 transposon from flatfish, is functionally active in vertebrate cells. Nucleic Acids Res. 2009;37:1239-47. 
40. Miskey C, Izsvák Z, Plasterk RH, Ivics Z. The Frog Prince: A reconstructed transposon from Rana pipiens with high transpositional activity in vertebrate cells. Nucleic Acids Research. 2003:6873-81.

41. Coy MR, Tu Z. Gambol and Tc1 are two distinct families of DD34E transposons: analysis of the Anopheles gambiae genome expands the diversity of the IS630-Tc1-mariner superfamily. Insect Mol Biol. 2005.

42. Muñoz-López M, García-Pérez JL. DNA transposons: nature and applications in genomics. Current genomics. 2010;11:115-28

43. Hua-Van A, Langin T. Daboussi MJ. Molecular Biology and Evolution: Evolutionary history of the impala transposon in Fusarium Oxysporum; 2001.

44. Platt RN, Mangum SF. Ray DA. Mobile DNA: Pinpointing the vesper bat transposon revolution using the Miniopterus natalensis genome; 2016.

45. Pujolar JM, Astolfi L, Boscari E, Vidotto M, Barbisan F, Bruson A, et al. Tana1, a new putatively active Tc1-like transposable element in the genome of sturgeons. Mol Phylogenet Evol. 2013;66:223-32.

46. Yang P, Craig PA, Goodsell D, Bourne PE. BioEditor-simplifying macromolecular structure annotation. Bioinformatics. 2003;19:897-8.

47. Xia X. DAMBE7: new and improved tools for data analysis in molecular biology and evolution. Molecular Biology \& Evolution. 2018;35:1550-2

48. McGuffin LJ, Bryson K, Jones DT. The PSIPRED protein structure prediction server. Bioinformatics. 2000;16:404-5.

49. Edgar RCBT-CSBC. MUSCLE: multiple sequence alignment with improved accuracy and speed. 2004.

50. Nicholas KB, Nicholas HB, Deerfield DWI. GeneDoc: Analysis and visualization of genetic variation, EMBNEW. Embnew News. 1997;4:págs. 28-30.

51. Kumar S, Stecher G, Tamura K. MEGA7: Molecular Evolutionary Genetics Analysis Version 7.0 for Bigger Datasets. Molecular Biology \& Evolution. 2016;33:1870.

52. Kalyaanamoorthy S, Minh BQ, Wong TKF, Von Haeseler A, Jermiin LS. ModelFinder: fast model selection for accurate phylogenetic estimates. Nat Methods. 2017:14:587-9.

\section{Publisher's Note}

Springer Nature remains neutral with regard to jurisdictional claims in published maps and institutional affiliations.

Ready to submit your research? Choose BMC and benefit from:

- fast, convenient online submission

- thorough peer review by experienced researchers in your field

- rapid publication on acceptance

- support for research data, including large and complex data types

- gold Open Access which fosters wider collaboration and increased citations

- maximum visibility for your research: over $100 \mathrm{M}$ website views per year

At $\mathrm{BMC}$, research is always in progress.

Learn more biomedcentral.com/submissions 